\title{
De l'image radar à la prévision des crues
}

\author{
From the radar image to the flood forecasting
}

\author{
par J.L. Roy \\ Direction de l'eau \\ avec les contributions de JJ Vidal, DIREN Midi Pyrénées et de M.-P. Nérard, Direction de l'eau
}

This paper deals with questions about the operational flood forecasting development perspectives taking into account experiences and research and the hydrometeorological radars progressive installation. These radars allow to know more about water waves. But the objectives of searchers and alert professionnals are not exactly the same and an active cooperation between scientifics and field professionals is necessary to progress.

\section{I — PRÉAMBULE}

L'annonce des crues s'est considérablement transformée ces dernières années mais va devoir encore évoluer fortement dans les années à venir. D'une part la demande sociale est toujours aussi présente, du moins après chaque événement grave, d'autre part, les outils ont progressé. La puissance des ordinateurs n'est plus un problème et la télétransmission des données est généralisée. Mais surtout sont progressivement mis en place de nouveaux outils, les radars hydrométéorologiques. Le public, les élus le savent et attendent donc un progrès.

La question est de savoir comment ces nouveaux outils vont permettre d'apporter une amélioration aux systèmes d'alerte, soit avec des délais plus importants et une précision plus grande, soit en permettant de développer l'alerte sur de petits bassins aux crues rapides.

Les facteurs limitant actuellement la progression de l'annonce vers un allongement des délais et vers la surveillance de cours d'eau supplémentaires à crues rapides, sont d'une part les méthodes, d'autre part l'organisation.

Cependant la question qui se pose pour l'instant n'est pas "qui va faire?", car la question "comment faire" n'a pas encore de réponse claire. Les questions liées à l'organisation vont devoir être abordées, mais seulement après qu'auront été mises au point des méthodes opérationnelles permettant d'utiliser au mieux les nouveaux outils.

On ne verra donc ici que les méthodes, en supposant qu'à chaque instant il y ait des hommes pour les mettre en œuvre, et d'autres hommes également prêts à agir sur la base des informations recueillies et calculées par les premiers. Mais il est clair que dès que les méthodes seront au point, se reposeront les questions d'organisation.
Peut-on dire aujourd'hui, ces questions étant supposées réglées (ainsi que les questions de financement, d'astreinte et de formation), que l'alerte aux crues rapides est possible ? Car c'est bien sur la prévision des crues rapides que des progrès sont attendus, pour alerter et informer, partout où vivent des personnes susceptibles d'être menacées par les eaux. Sur le plan des techniques et des méthodes, qu'est-ce qui empêche de développer des systèmes d'annonce et d'alerte sur les petits bassins à crues rapides ? Peut-on fixer des échéances ? D'ores et déjà l'utilisation qualitative des images radars permet aux professionnels de l'alerte de voir l'événement dans sa globalité et dans son évolution, alors qu'auparavant, l'information ne filtrait que de manière discontinue à partir de quelques points disséminés sur un bassin. Mais quand passera-t-on au stade quantitatif ?

\section{DÉFINITIONS}

Avant d'aller plus loin quelques définitions :

Qu'est-ce que la prévision? Ce mot est entendu de deux manières.

Pour le chercheur hydrologue, la recherche permet de comprendre les phénomènes, d'expliquer et de décrire leur déroulement. Quand on sait le faire on peut prévoir l'issue d'une situation, mais cette prévision n'est émise que dans le but de vérifier que l'on a bien compris et bien expliqué le processus, que la théorie, les équations, les données... sont bonnes. Ce travail est fait en temps différé sans pressions extérieures, sans obligation de réponse immédiate sur les niveaux et les délais. Quand le chercheur constate que son explication est bonne, c'est-à-dire qu'elle permet de bien expliquer et donc prévoir l'évolution du phénomène, il a fait son travail. 
Pour le professionnel de l'alerte, la prévision n'est pas un objectif de recherche, c'est un objectif opérationnel pour déclencher des actions. C'est une information qu'il doit produire sur un événement futur avec le maximum de précision et de délai, pour la transmettre afin de déclencher à bon escient des actions de mise en sécurité des personnes. Pour lui c'est la fiabilité du résultat en temps réel qui compte. Ces deux conceptions coexistent. Elles doivent se rapprocher si l'on veut espérer des progrès dans ce domaine. Il est essentiel que chercheurs et prévisionnistes travaillent ensemble. Qu'est-ce qu'une crue rapide ?

On parle de temps de concentration de quelques heures, généralement de 12 heures entre la pluie et la pointe de la crue. C'est une valeur arbitraire qui ne correspond pas à une limite physique. Les crues rapides sont les crues des petits bassins au relief marqué, de quelques centaines de $\mathrm{km}^{2}$. Partout où les temps de concentration sont de l'ordre de la dizaine d'heures, dispose-t-on de méthodes permettant d'annoncer le dépassement d'une côte dangereuse de manière efficace avec une avance suffisante, c'est-à-dire permettant de déclencher les actions de mise en sécurité nécessaires ? Le chercheur hydrologue répondra plutôt de manière positive, le responsable de service opérationnel d'alerte sera plus réservé. Pourquoi ?

\section{Q QUE SAIT-ON ? QUE SAIT-ON FAIRE ?}

Le chercheur hydrologue connaît les théories, les travaux de recherche. On sait interpréter le signal radar, traiter les problèmes d'échos de sol, de masques, de bande brillante. On peut donc évaluer avec précision et finesse les lames d'eau précipitées.

En pratique se posent certaines questions : Est-ce à l'utilisateur de traiter complètement l'image radar transmise pour la transformer en lame d'eau, ou bien est-ce une lame d'eau distribuée prête à l'emploi, traitée avant livraison qui lui est nécessaire? Comment se partagent les responsabilités respectives attachées à la constitution de l'image brute, au calcul de la lame d'eau et enfin à son utilisation ?

Comment est-il le plus judicieux de traiter cette image brute pour en tirer une lame d'eau ? Par recalage en temps réel à partir d'un petit nombre de pluviographes, ou sur la base d'un calage a priori obtenu à partir des mesures effectuées lors d'épisodes antérieurs au moyen d'un grand nombre d'appareils ? Ne pourrait-on envisager de rendre complémentaires ces deux méthodes ? Sinon il est impératif de pouvoir expliquer en quoi elles sont incompatibles et dans quelle configuration l'une est mieux adaptée que l'autre.

Comment traiter au mieux les effets de masques ? Par traitement du signal provenant de la zone masquée ou par advection des pixels voisins non masqués ?

Supposons acquise la lame d'eau, par l'un ou l'autre procédé, que fait-on de ces valeurs de pluie brute finement distribuées sur l'ensemble d'un bassin ?

On cherche à évaluer la part de cette pluie qui va contribuer à brève échéance à la variation rapide des débits au point exutoire, et à lier la pluie dite efficace à cette variation de débit pour prévoir cette dernière (dans les deux sens du terme prévoir que nous avons vus ci-dessus).

La difficulté est qu'on ne sait pas mesurer directement une pluie efficace, celle qui ruisselle sans attendre et qui rejoint au plus vite l'exutoire. On transforme la donnée pluie brute en une donnée intermédiaire non mesurable que l'on transforme à son tour en un volume qui s'écoule un peu plus tard à l'exutoire et à qui on donne une certaine forme, ce qui donne le débit qui lui, est mesurable.

Il y a plusieurs méthodes et plusieurs voies de recherche, mais aussi plusieurs objectifs.

Quelles que soient ces méthodes, la seule vérification possible de leur validité, c'est le résultat final : le débit sortant, même si des mesures intermédiaires peuvent apporter des indices. Donc à l'entrée une pluie que l'on sait évaluer avec précision dans son intensité et sa distribution, à la sortie un débit. Entre les deux, l'action d'un bassin versant, complexe, dépendant de multiples paramètres.

Des types de modèles pluie-débit, il en existe de plusieurs sortes, à condition bien sûr de disposer de suffisamment de données d'événements réels pour les caler : modèles statistiques, déterministes, à réservoirs, hydrogramme unitaire, isochrones, distribués, DPFT...

Mais en opérationnel pour les crues rapides, ils ne marchent pas très bien. Même si le résultat est bon sur une partie des événements de calage, il peut ne pas l'être sur tous. Les niveaux prévus peuvent être corrects, avec un écart important sur les délais, ou l'inverse. En fait on ne peut pas dire qu'il existe actuellement de modèle vraiment opérationnel utilisé par les services pour la prévision des crues rapides. Et pourtant quand le professionnel de l'alerte regarde son écran et qu'il voit la pluie, son intensité, sa répartition et son déplacement, il pressent que, à quelques dizaines de kilomètres à l'aval, à l'exutoire, le niveau de la rivière va bientôt monter, et très vite. Alors il prend son téléphone et déclenche l'alerte.

Qu'est-ce que les modèles apportent de plus ? un hydrogramme à quelques heures pas toujours bon. Pour gérer une retenue à niveau à peu près constant avec une revanche de sécurité pour stocker les erreurs de prévision, ça peut encore aller et encore à condition que ce ne soit pas une retenue dont la fonction est l'écrêtement des crues, mais pour déclencher une alerte, c'est plus troublant.

Le problème est que toute la richesse d'information que le prévisionniste peut lire sur son écran est uniformisée par la plupart des modèles, moyennée sur le bassin versant ou à la rigueur sur quelques sous-bassins, parce que les modèles pluies-débits sont construits sur la base d'une correspondance entre lames d'eau moyennes précipitées et débits à l'exutoire.

Le modèle mélange l'information que le radar, les pluviographes et les algorithmes de calcul ont permis de dessiner finement. C'est comme si on réduisait un tableau de Van Gogh à quelques grosses taches de peinture rouge, bleue, jaune ou verte.

Avec une dizaine de pluviographes on extrapole l'information en supposant que chaque appareil représente un territoire qui lui est plus ou moins arbitrairement affecté (Thiessen, fonctions Spline, krigeage...). Avec un radar équivalent de plusieurs milliers de pluviographes, on réduit l'information à celle que donneraient une dizaine de pluviographes, certes plus représentatifs que les vrais, mais cela n'en fait toujours qu'une dizaine. En outre les modèles ayant souvent été calés avec des mesures de pluies au sol, l'introduction de pluies radar moyennées sur des secteurs équivalents ne peut que créer des écarts supplémentaires. Si le gain qualitatif est évident (visualisation de l'événement), on ne peut pas encore parler de gain quantitatif. 
Un radar vaut le prix de quelques centaines de pluviographes mais c'est l'équivalent physique de plusieurs milliers de pluviographes. C'est donc a priori intéressant. Mais pour calculer des prévisions à partir de l'image radar, on réduit l'information recueillie à ce que donnerait une dizaine de pluviographes. Certes, on utilise l'image de manière qualitative, mais il est clair qu'il faut progresser vers une utilisation plus quantitative valorisant pleinement l'information recueillie et les investissements effectués.

Deux objectifs : La description correcte des phénomènes, d'une part, le déclenchement d'alertes à bon escient d'autre part. Pour les deux objectifs on peut chercher à construire l'hydrogramme sortant en prenant en compte de manière plus ou moins fine l'action intermédiaire du bassin : ETP, infiltration, détermination des zones contributives, écoulement souterrain, propagation, en découpant le bassin en sous-bassins de plus en plus petits, pour arriver à la limite jusqu'à essayer de suivre ce que devient la pluie de chaque pixel. On peut aussi partir de chaque pixel et les regrouper en fonction de considérations liées à la nature du sol, à la végétation, à la pente, à la saturation du sol etc.

Pour le second objectif, celui de l'alerte, on peut chercher plutôt qu'un hydrogramme, à établir des relations entre la configuration des pluies (répartition, intensité) et les variations de débit à l'exutoire, c'est-à-dire à construire des indicateurs qui ne donneront pas la valeur des débits et des hauteurs, mais qui diront si il y a danger ou non.

Nous verrons plus loin quelques exemples de recherches ou de démarches en cours suivant ces différentes approches. Quelques questions : sur les modèles distribués, faut-il seulement attendre une accumulation suffisante de données radar pour les caler ? Y a-t-il encore des barrières théoriques à franchir ? La multiplicité des variables génère-t-elle des incertitudes croissantes dont l'importance finit par compenser le gain obtenu par la discrétisation de l'information ? Quelle est l'opinion des chercheurs spécialistes sur ces questions ? Il serait utile de le savoir pour orienter les investissements de la manière la plus efficace, ou pour pouvoir dire clairement que dans certaines zones à risques les solutions sont ailleurs que dans l'alerte.

Enfin, pour terminer sur les questions, la nécessaire prise en compte dans les systèmes informatiques opérationnels des diverses avancées méthodologiques ne peut-elle pas être freinée par la structure de ces derniers ?

\section{IV — BILAN NON EXHAUSTIF DES EXPÉ- RIENCES RÉCENTES OU EN COURS}

\subsection{Etude crues éclairs}

Cette étude a été réalisée d'août 1996 à fin 1998, par le LHF, RHEA et EDF, la direction de l'eau étant maître d'ouvrage. L'objectif était de tester la faisabilité d'un outil opérationnel pour la prévision des crues rapides, en réalisant un outil prototype. Il ne s'agissait pas de créer de nouveaux éléments mais d'associer des modules existant "sur le marché". En cas de succès dans la réalisation du prototype, l'outil devait ultérieurement être rendu convivial puis proposé aux services d'annonces des crues.

L'un des intérêts de cette démarche dont nous allons dresser un bref bilan est qu'elle a permis de réunir périodiquement autour de ce projet professionnels de l'annonce et chercheurs. Elle a également mis en évidence la nécessité de structurer de manière très rigoureuse le recueil et l'archivage des données sans lesquelles toute tentative de modélisation est vaine.

\subsubsection{Description sommaire de l'outil prototype crues-éclairs}

Il s'agissait d'enchaîner complètement le processus de l'image à la prévision en utilisant l'outil CALAMAR de RHEA pour le calcul des lames d'eau, le modèle DPFT d'EDF pour la prévision et la plate-forme MISTERE du LHF pour l'intégration et l'exploitation des outils de prévision avec notamment une procédure multimodèle, en créant un outil de simulation sur lequel des prévisionnistes de services d'annonce des crues pourraient effectuer des tests.

Deux sites ont été retenus pour effectuer les tests : le Gardon d'Anduze dans le Gard et le Lignon du Velay en Haute-Loire (critères physiques et morphodynamiques, bassins peu aménagés, couverture radar, données disponibles, existence de modélisation, risques d'inondation).

Le calage des différents modèles DPFT a été effectué à partir de données sol, les simulations étant réalisées sur images radar. Les bassins ont été traités de manière globale, ou bien divisés en deux sous-bassins, mais on n'a pas utilisé la distribution fine de la pluie

Enfin des indicateurs de risque de dépassement de seuils d'alerte ont été testés.

\subsubsection{Bilan de l'étude}

Il ressort nettement de l'expérience la nécessité d'une part de spatialiser la modélisation en découpant le bassin, et d'autre part de travailler à pas de temps plus fins, afin de tester ce qu'on pourrait gagner en précision. La prévision de la pluie peut permettre de gagner environ une heure sur les délais de prévision.

Une partie très instructive a été la phase de tests de simulation sur l'outil à laquelle ont travaillé une douzaine d'agents de services d'annonce de crues pendant près de deux mois. Les agents ont parfois été plus troublés qu'aidés par les résultats des modèles dans la mesure où ceux-ci ne leur apportait pas systématiquement des éléments d'information fiables pour déclencher à coup sûr des alertes.

Il est clair également que la prévision des crues rapides ne peut être mise en œuvre que par des personnels expérimentés et formés à l'interprétation des résultats, qui sont un élément de décision mais pas le seul.

Il est indispensable que l'outil présente une très bonne ergonomie et convivialité, pour éviter toute perte de temps dans la prise de décision.

En conclusion on retiendra l'avis exprimé par les agents : l'apport de l'imagerie radar calibrée est mis en avant par la sécurisation qu'il procure grâce à la visualisation spatiale de l'événement et par la confirmation de la gravité de l'événement qu'il permet de mettre en évidence. Mais les modèles utilisés en ne prenant pas en compte la répartition spatiale de la pluie dégradent la richesse des données radar. Il paraît nécessaire de s'orienter vers l'utilisation de modèles distribués. En outre le système doit être un outil d'aide à la décision et ne pas contribuer à multiplier les questions qui se posent au prévisionniste en lui proposant trop de scénarios. Cette expérimentation a montré que la prévision des crues rapides nécessitait encore quelques préalables avant d'entrer dans une phase opérationnelle : accumulation de données, constitution d'outils ergonomiques et conviviaux, mais aussi 
mise au point de fonctions de transformation pluies-débits valorisant pleinement les données radar distribuées. Faut-il n'envisager qu'à long terme l'idée de prévoir des hydrogrammes et s'attacher plus activement à la détermination d'indicateurs binaires destinés à déclencher des alertes sans information fine associée ?

\subsection{Projet ALHTAIR de la DDE du Gard (ALarme Hydrologique Territoriale Automatisée par Indicateur de Risque)}

Ce projet constitue une suite à l'étude crues-éclairs. Il a pour objet de construire un indicateur d'alerte à partir de la mesure radar pixelisée. Le but est de prévoir la rapidité de la montée des eaux, le moment du maximum et de pouvoir qualifier l'ampleur des submersions à attendre. La pluie brute est transformée en pluie efficace par prise en compte de l'infiltration calculée au pixel sur la base de la loi de Horton. A partir d'un modèle numérique de terrain, la distance hydraulique de chaque pixel à l'exutoire a été déterminée. Ces distances sont ensuite transformées en temps de propagation à l'exutoire sans prise en compte des phénomènes d'interférences ou de stockage intermédiaire.

Les résultats obtenus sont encourageants. La DDE du Gard poursuit l'étude et l'expérimentation et souhaite s'associer en partenariat avec d'autres organismes ayant des objectifs similaires. Il est effectivement souhaitable qu'un groupe de suivi associant services et chercheurs se constitue autour de ce projet.

\subsection{Etude conduite par la DIREN Centre} (avec la société RHEA)

Il s'agit là aussi d'une suite de l'étude crues-éclairs, ayant pour but d'intégrer au système CALAMAR des indicateurs de risques hydrologiques sur de petits bassins affluents de la Loire, établis pour les épisodes importants, par régression inverse entre les débits en phase de montée et les pluies horaires antérieures, et destinés à aider les services d'annonce des crues dans leur prise de décision.

\subsubsection{Système VALERHY (Météo France et DIREN Midi Pyrénées)}

Un second outil de calcul et de visualisation des lames d'eau précipitées va entrer en phase opérationnelle. C'est le système VALERHY développé par Météo France, qui capitalise les travaux de recherche sur le traitement des images radar réalisés dans le cadre du groupement GISELE (Météo France, Laboratoire d'Aérologie de l'université de ToulouseRangueil et la DIREN Midi-Pyrénées). Le système SOPHIE, développé par la DIREN, intègre directement les valeurs ainsi calculées pour l'établissement de prévision de débits. La DIREN poursuit des études en coopération avec l'Institut de Mécanique des Fluides de Toulouse pour le développement de méthodes utilisant l'information spatialisée contenue dans les images radar.

\subsubsection{Thèse de Delphine Grelat (en cours à la DIREN Centre)}

Cette étude vise à comprendre le fonctionnement hydrologique des bassins versants contributifs amont de la Haute Loire, notamment en matière de production (passage de la pluie brute à la pluie nette), par le biais d'un modèle déterministe utilisant les entrées météorologiques (pluie sol et ETP) et les caractéristiques physiques (topographie, végétation, propriétés des sols...).
Le calage et la validation de la représentation du fonctionnement hydrologique par le modèle est d'abord effectuée sur un bassin versant bien connu (Lignon du Velay) avant d'évaluer la transposition sur d'autres bassins versants physiquement proches mais non ou peu jaugés.

$\mathrm{Si}$ les résultats sont satisfaisants, la connaissance du fonctionnement hydrologique de ces sous-bassins contributifs pourrait permettre l'amélioration des méthodes de prévision, par une prise en compte plus précise des apports intermédiaires.

Par ailleurs, le modèle déterministe permet d'effectuer des tests de sensibilité à l'introduction d'une pluie spatialisée telle qu'elle est fournie par le traitement CALAMAR des images radar.

Enfin, une comparaison avec une approche semi-distribuée plus classique doit permettre d'évaluer les avantages et les limites de ces modèles, notamment en utilisation opérationnelle.

\subsubsection{Thèse de Rachel Datin (LTHE)}

Cette thèse soutenue le 23 octobre 1998 s'articule autour du projet Ardèche, mené par EDF, Météo France et le LTHE, pour évaluer les capacités de prévision possibles sur les petits bassins à crues rapides en testant, sur une zone correctement instrumentée, la faisabilité et la viabilité d'un outil opérationnel de prévision hydrométéorologique des crues subites.

Le travail est mené sur la base de lames d'eau moyennes calculées soit par mesures au sol soit par images radar. La fonction de production est fournie par le modèle TOPMODEL (détermination des zones contributives liées aux remontées de nappes, prise en compte d'un débit d'exfiltration de la nappe). Dans ce travail est examinée avec attention l'erreur générée par l'incertitude sur l'évaluation des lames d'eau moyennes à partir de mesures ponctuelles de pluie, et dont les conséquences peuvent être très importantes sur les débits prévus.

Un générateur de pluies futures intégrant la pluie antérieure, la pluie prévue dans les deux heures et les prévisions de pluie sur 24 heures, a été construit et couplé avec le modèle de prévision de manière à fournir une gamme d'hydrogrammes prévus assortis de probabilité.

Enfin, une évolution du modèle a été proposée pour intégrer l'information spatialisée sur l'entrée pluie. Là aussi les résultats sont encourageants, mais des études et recherches complémentaires restent indispensables avant de parvenir à un outil réellement opérationnel.

\section{$\mathrm{V} \square$ CONCLUSION}

Ces quelques exemples, non exhaustifs, montrent que les initiatives sont diverses et les approches variées tant pour la compréhension des phénomènes que pour la mise au point d'outils opérationnels.

La conclusion de cet exposé, sans doute simplificateur et surtout constitué de questions, c'est qu'il ne paraît pas déraisonnable d'envisager de sérieux progrès dans les prochaines années pour l'alerte opérationnelle aux crues rapides, avec l'accumulation des données radar et la consolidation progressive des méthodes. Mais cela ne sera pas possible sans une coopération active et soutenue entre le monde de l'hydrologie scientifique et celui de l'alerte opérationnelle. 